\title{
FATORES MOTIVADORES PARA A FORMAÇÃO DE ALIANÇAS ESTRATÉGICAS ENTRE COOPERATIVAS MINEIRAS: UM ESTUDO DE CASO
}

Sara da Costa Fernandes ${ }^{1 *}$, Jamille Araújo Paixão², Vagner Rosalem³.

${ }^{*}$ Autor para correspondência

1. Mestranda em Gestão Organizacional, Universidade Federal de Goiás (UFG),

Campus Catalão. Catalão - GO, Brasil. (sara.scf1@gmail.com)

2. Mestranda em Gestão Organizacional, Universidade Federal de Goiás (UFG),

Campus Catalão. Catalão - GO, Brasil.

3. Doutor em Administração de Empresas, EAESP/FGV; Docente na

Universidade Federal de Goiás (UFG), Campus Catalão. Catalão - GO, Brasil.

Recebido em: 03/10/2016 - Aprovado em: 21/11/2016 - Publicado em: 05/12/2016

DOI: 10.18677/EnciBio_2016B_187

\begin{abstract}
RESUMO
Diante das mudanças estruturais econômicas ocorridas nos últimos anos, as organizações precisam redefinir suas estratégias em busca de melhor posicionamento de mercado. No setor agroindustrial, as cooperativas possuem importância econômica e social dado que agregam valor à produção rural em muitas regiões. Ao considerar a produção agropecuária cooperativista em vários estados brasileiros, as cooperativas do Estado de Minas Gerais se destacam pela capacidade produtiva e possuem maior representatividade no cenário nacional. Em busca de maior eficiência produtiva, combinações de ideias existentes, capacidades, habilidades e recursos, as cooperativas adotam as alianças e parcerias estratégicas como forma de manter a sobrevivência. Esta pesquisa teve como objetivo identificar os principais fatores que determinam a formação de aliança estratégica entre cooperativas do Estado de Minas Gerais. A pesquisa foi realizada por meio de um estudo de caso, cujo resultado contribui para a compreensão e importância do relacionamento entre cooperação e alianças estratégicas entre organizações através da união de forças, superação de entraves e aproveitamento de oportunidades do setor agroindustrial do Estado Mineiro.
\end{abstract}

PALAVRAS-CHAVE: Alianças estratégicas, Cooperativas, estratégias.

\section{MOTIVATORS FACTORS TO CREATE STRATEGIC ALIANCES BETWEEN MINAS GERAIS STATE COOPERTATIVES}

\section{ABSTRACT}

Faced with economic structural changes in recent years, organizations must redefine their strategies to search a better market placement. In the agribusiness, the cooperatives have economic and social matters, because they adds value to the rural production in several regions. Considering the cooperative rural production in several Brazilian states, the cooperatives in Minas Gerais State stand out for their productive capacity, and are considered the most representative on the 
national scene. In search of greater productive efficiency, combinations of existing ideas, capacities, skills and resources, the cooperatives are adopting the strategic alliances and partnerships in order to maintain their operations. This research aimed to identify the main factors that determine the formation of strategic alliance between cooperatives of the Minas Gerais State. The research was conducted through a case study, whose the findings contribute to the understanding and importance of the cooperation relationship and strategic alliances between organizations by joining forces, overcoming obstacles and taking advantage of opportunities in agribusiness production of the Minas Gerais State.

KEYWORDS: Strategies, Cooperatives, Strategic alliances.

\section{INTRODUÇÃO}

O modelo econômico do mundo capitalista estabelece novas tendências de mercado de modo que as organizações passam a atuar em um mercado dinâmico, marcado pelo contínuo processo de globalização que exije conquistas de vantagens competitivas e sobrevivência (SOUZA \& BALDIM, 2006). Diante das mudanças estruturais que têm ocorrido nos últimos anos, tais como a alteração das formas tradicionais de intervenção do Estado e desregulamentação do mercado, houve a reestruturação produtiva e a subsequente alteração do padrão de competitividade entre as organizações. No setor agroindustrial, esta situação foi refletida na redefinição das estratégias empresariais para processos de desenvolvimento a partir de novas formas de organização, dentre elas as cooperativas agropecuárias (PELEGRINI et al., 2015).

Segundo LAGO (2009), as cooperativas agropecuárias apresentam-se "como uma forma de organização da produção e coordenação de sistemas agroindustriais" na busca por eficiência produtiva e vantagem competitiva. PELEGRINI et al. (2015) apontam que o avanço do cooperativismo agropecuário tem papel estratégico na economia e para o desenvolvimento de políticas de desenvolvimento rural. Além disso, promove a inserção de pequenos e médios produtores no mercado, permitindo a ampliação do acesso ao desenvolvimento social em regiões isoladas no país.

Considerando o setor produtivo em vários Estados brasileiros, o Estado de Minas Gerais se destaca na produção agropecuária através do aumento da capacidade produtiva, pelo uso intenso de tecnologias, incentivos a pesquisas e aumento da profissionalização dos trabalhadores. Esse Estado é considerado,então, um dos Estados brasileiros com maior representatividade no cenário nacional (SOUZA, 2010).

As cooperativas do Estado de Minas Gerais são as organizações que mais refletem em mudanças estruturais no setor agropecuário em prol da competitividade. Conforme as informações do Sindicato e Organização das Cooperativas do Estado de Minas Gerais (OCEMG), as cooperativas no Estado representam 11,1\% das 7.143 cooperativas brasileiras e possui participação anual de 6,4\% no PIB do Estado. Especificamente, a concentração e participação cooperativista na produção agropecuária em Minas Gerais ocorrem nas regiões do Triângulo Mineiro e Alto da Parnaíba, Sul e Noroeste de Minas (SILVA et al., 2012).

LAGO (2009) apresenta que novas estratégias de posicionamento de mercado e cooperação são elementos predominantes para as cooperativas agropecuárias através de combinações de idéias existentes, capacidades, habilidades e recursos. Para isto, as cooperativas passaram a atuar em redes de cooperação através da formação de alianças ou parcerias estratégicas. 
As alianças estratégicas consistem em um processo de cooperação entre organizações, que de forma colaborativa compartilham estratégias e recursos, visando potencializar competências e alcançar os objetivos estratégicos das respectivas organizações (FAÇANHA, 2012). A parceria interorganizacional "tem sido base para o sucesso de muitas organizações, pois são nestas relações de parceria e cooperação que se pode aproveitar as capacidades individuais de forma coletiva" (LAGO, 2009).

A importância em compreender o relacionamento de cooperação e alianças estratégicas entre organizações do setor agroindustrial, considerando o Estado de Minas Gerais como uma das regiões mais significativas para a produção agropecuária no Brasil, é a base deste estudo. Ademais, as cooperativas agropecuárias são expressivos atores na cadeia produtiva e possuem influência na geração de emprego, renda, exportações e estabilização de preços da economia (BARROS, 2006).

Esta pesquisa teve como objetivo principal identificar os principais fatores motivadores que determinam a formação de uma aliança estratégica entre cooperativas do Estado de Minas Gerais. Para atingir este objetivol, este estudo teve como objetivos específicos identificar o tipo de aliança estratégica estabelecida entre as cooperativas do Estado Mineiro, e avaliar quais modificações já ocorreram como resultado da aliança estratégica nas cooperativas estudadas.

\section{MATERIAL E MÉTODOS}

Visando atingir os objetivos propostos, de explorar e descrever os motivos prováveis para a formação da parceria e aliança entre cooperativas, efetuou-se um estudo de caso único, de natureza qualitativa, envolvendo seis cooperativas agropecuárias localizadas em Minas Gerais.

Com um caráter exploratório e natureza descritiva, foi feito um levantamento das informações a seguir: quais os fatores motivaram as cooperativas a trabalharem na forma de parcerias, o porquê da escolha por essa forma de organização, em qual momento iniciou o processo de cooperação, quais são as características e o tipo da aliança estratégica formada entre elas, qual é o faturamento anual das cooperativas, e como é composta a estrutura e a governança corporativa do consórcio entre cooperativas.

A coleta de dados foi realizada em Agosto de 2016, e ocorreu por meio de entrevistas semi-estruturadas com o diretor executivo do consórcio das cooperativas e com coordenadores executivos das cooperativas envolvidas no processo de aliança estratégica. Considerando 0 universo da cadeia produtiva das cooperativas, cabe ressaltar o acesso às pessoas e aos dados. Foram também levantados dados secundários extraído de documentos e relatórios relacionados aos fatores que motivaram a parceria entre as cooperativas agropecuárias. Foi apresentada a análise documental de projetos.

Para condução da análise de dados a presente pesquisa utilizou a técnica de análise de conteúdo, que refere-se a um conjunto de técnicas de análise de comunicação que dispõe informação através de uma fonte, permitindo gerar conhecimentos da produção de mensagens seguindo procedimentos que descrevem o conteúdo das entrevistas e documentos analisados (BARDIN, 2009). Os dados foram organizados, consolidados em grupos de acordo com as características das informações, e foram interpretados por meio da síntese e relacionamento entre eles.

Foram levantados e analisados dados primários (entrevistas) e secundários 
(documentos) referente à formação de aliança estratégica entre as cooperativas envolvidas, como fonte de evidências e informações, possibilitando a identificação de fatores motivadores para a formação da parceria e o tipo de aliança estabelecida.

\section{RESULTADOS E DISCUSSÃO}

O Consórcio Cooperativo Grupo Cemil - CCGC é uma aliança estratégica entre cooperativas do ramo agropecuário presentes no Alto Paranaíba, Noroeste e Centro Oeste do Estado de Minas Gerais. Este consórcio conseguiu se estabelecer ao unir forças de seis consorciadas que operam no segmento de leite e café, sendo estas: Cooperativa Agropecuária de Carmo do Paranaíba - Carpec, Cooperativa Central Mineira de Lacticínios - Cemil, Cooperativa Mista Agropecuária de Dores do Indaiá - Comadi, Cooperativa Agropecuária de Patrocínio - Coopa, Cooperativa Mista Agropecuária de Patos de Minas - Coopatos, Cooperativa Agropecuária do Vale do Paracatu - Coopervap, conforme Figura 1.

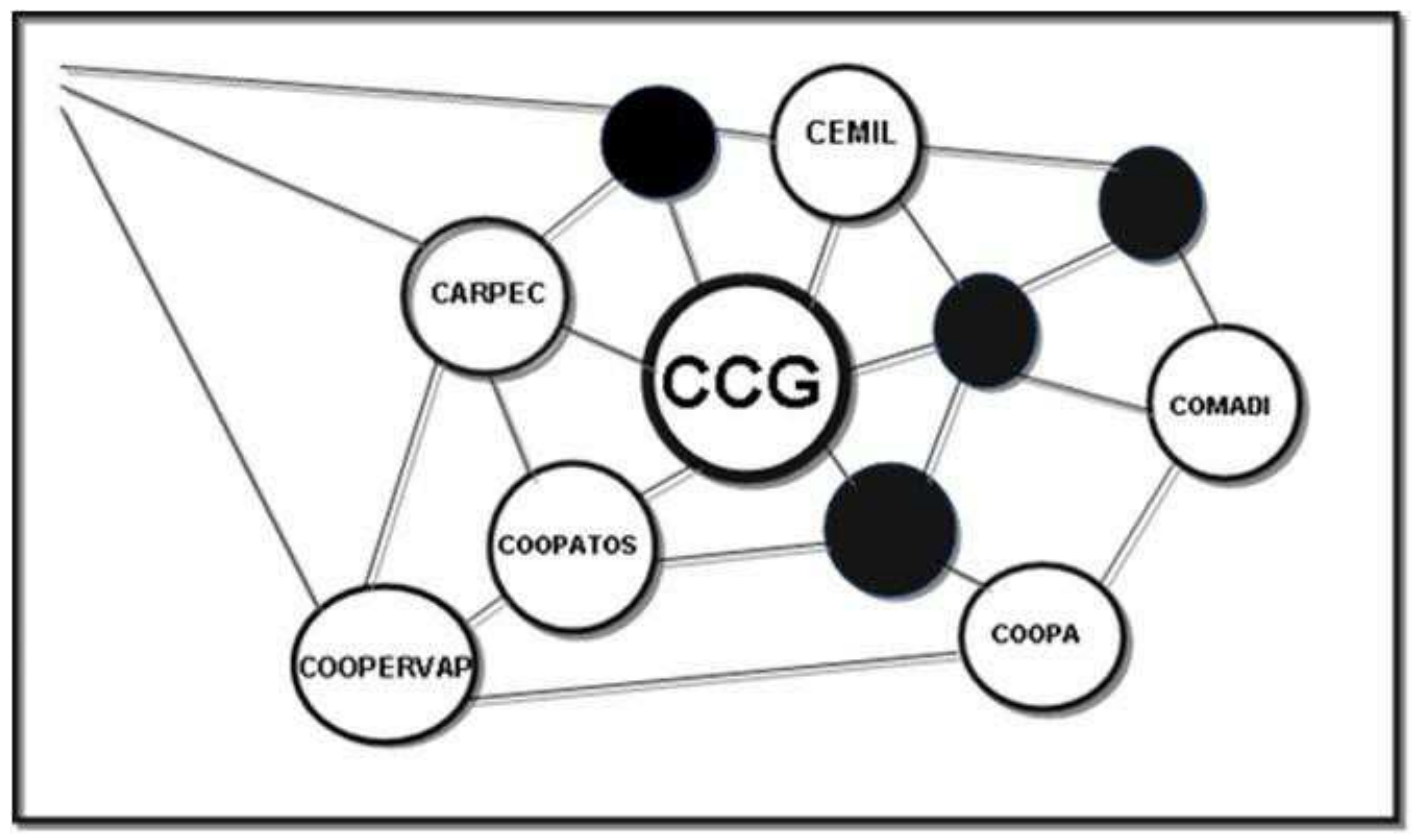

FIGURA 1 - Formação do Consórcio Cooperativo Grupo Cemil.

FONTE: Elaborado pelos autores, a partir de informações do site do CCGC (2016)

O consórcio iniciou suas atividades em 2013 e juntas estas cooperativas conseguem atender mais de 50 municípios mineiros, além de formarem um quadro social composto por 9.000 associados. Esta intercooperação começou a se estabelecer através de uma busca incessante de praticar os princípios cooperativistas; para isto, iniciaram as atividades através da formação de uma central de compras, a qual movimentou nestes últimos três anos, mais de $R \$$ $600.000 .000,00$ (Seiscentos milhões de reais).

A estratégia de cooperação adotada pelas seis cooperativas é uma alternativa encontrada por diversas organizações como resposta aos estímulos empresariais da atualidade. Estas iniciativas visam criar e manter vantagens competitivas de cada um dos participantes por meio da otimização dos custos e sinergia com outros parceiros que possuem algum diferencial no mercado em que atuam. De acordo com o atual Diretor Executivo do CCGC a finalidade consiste na união de forças na busca pela superação de entraves e também o aproveitamento de oportunidade que ENCICLOPÉDIA BIOSFERA, Centro Científico Conhecer - Goiânia, v.13 n.24; p.1834 2016 
de maneira individual seria impossível de ser alcançada. Assim, estes são os fatores motivacionais predominantes para a formação da aliança estratégica entre as seis cooperativas mineiras, obtendo desta forma os benefícios da intercooperação e sustentabilidade através de um Consórcio Cooperativo.

Tomando por base as afirmações de ABBADE (2009), fica claro que as ações desenvolvidas por estas cooperativas para a formação desta aliança estratégica são necessidades explícitas em função da competitividade cada vez mais acirrada nos mercados. Desta forma, os mercados cooperativistas não se excluem, ao contrário, diante de suas peculiaridades como fins sociais e conselho administrativo, apresentam a necessidade latente de se adequarem a esta concorrência e buscarem soluções urgentes para sua manutenção e expansão.

Conforme os tipos de alianças estratégicas apresentadas por LORANGE \& ROSS (1996), o CCGC constitui-se como um empreendimento cooperativo formal, uma vez que possui a formalização desta parceria, além de uma estrutura administrativa formada por diretoria e funcionários. Atualmente o CCGC Corporativo possui um diretor executivo e uma controller, além da central de compras formada por cinco compradores sênior. Hoje, esta estrutura organizacional enxuta atende as necessidades do consórcio, uma vez este se formou há poucos anos e apresenta como destaque entre suas atividades a Central de Compras que envolve aquisição de produtos e serviços segmentados nas áreas: agropecuária, medicamentos, laticínio, logística, nutrição animal e supermercado para as cooperativas consorciadas.

O empreendimento busca a aglutinação de operações comuns para o alcance de ganhos coletivos, mas, não interferindo nas ações comerciais já desenvolvidas por qualquer uma das cooperativas participantes do consórcio. Neste contexto, as organizações saem do isolamento da compra individualizada e passam a obter vantagens da compra realizada por um montante maior. Os pedidos são faturados para cada cooperativa, entretanto, o volume total da negociação permite melhores preços e condições. Isto não interfere em outro tipo de ação comercial que seja desenvolvida por qualquer organização participante. $O$ atual Diretor Executivo do CCGC pontua que esta parceria traz benefícios para todos os integrantes, ratificando a execução dos valores e princípios cooperativistas, além de reforçar a imagem das integrantes com base na ética e transparência, ressaltando a marca de produtos e serviços em busca de um desenvolvimento organizacional mais sustentável.

No sistema cooperativista o indivíduo assume papel fundamental. Desta forma os comitês educativos e as assembléias são fontes de informação e discussão das ações propostas e desenvolvidas pela cooperativa através desta aliança estratégica. Apesar das seis cooperativas seguirem os mesmos princípios cooperativistas, cada instituição deve manter seus cooperados informados sobre suas ações estratégicas e adequá-las a realidade de cada região onde atuam, cumprindo assim o segundo princípio cooperativista, da gestão democrática.

Os profissionais que fazem parte do consórcio foram cuidadosamente selecionados entre as cooperativas, conveniadas com o objetivo de preservar 0 conhecimento, mas também, garantir a cultura cooperativista. Esta cultura também se mantém na formação da estrutura de Governança Corporativa, organizando as instâncias de maneira idêntica às cooperativas, apresentando apenas como diferencial a obrigatoriedade de troca anual e rotativa do Presidente do Conselho de Administração, conforme Figura 2. 


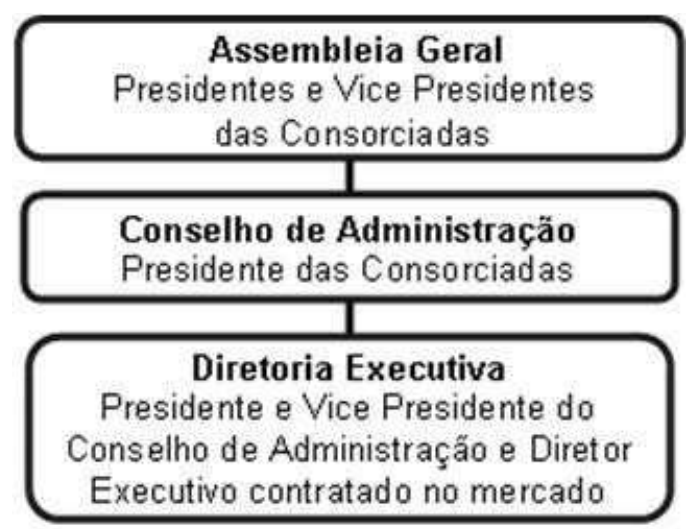

FIGURA 2 - Estrutura de Governança Corporativa CCGC.

FONTE: Elaborado pelos autores, a partir de informações do site do CCGC. (2016)

O estabelecimento da aliança estratégica propiciou um desenvolvimento planejado e com estrutura para suportar as alterações advindas do crescimento. Este avanço como FERREIRA \& BRAGA (2004) pontuam traz uma série de benefícios para toda a cadeia do agronegócio, além de melhorias sociais, uma vez que este tipo de empreendimento cooperativista colabora para a sustentabilidade de todos os envolvidos no processo. Os avanços advindos do setor cooperativista como um todo e principalmente a colaboração das cooperativas agropecuárias mineiras interferem diretamente no desenvolvimento do país e produzem melhorias para toda a cadeia produtiva na qual estão inseridas, reforçando assim sua importância não apenas local, mas nacional (SOUZA, 2010). Assim, mesmo diante das dificuldades para aplicação de uma aliança estratégica, esta se constitui como uma das alternativas para suportar os entraves econômicos e alterações constantes no mercado.

Segundo a visão apresentada pelo Coordenador Executivo da Cooperativa Agropecuária do Vale do Paracatu - Coopervap, as cooperativas de uma forma geral têm objetivos diferentes entre si e no caso específico da aliança estratégica para a criação do CCGC houve a necessidade de um posicionamento estratégico das cooperativas em razão da competitividade de mercado principalmente na aquisição de bens de produção e maior integração competitiva deste mesmo mercado. A aliança estratégica afetou de forma positiva com referência â competitividade maior em negociar bens de produção e melhores preços dos produtos Coopervap no mercado.

O que afeta negativamente são alguns aspectos culturais diferentes entre as integrantes, principalmente no comportamento e gestão das pessoas envolvidas. Entretanto, a colaboração entre as cooperativas é constante, principalmente no fator relativo à troca de conhecimentos relacionados às medidas legais tanto no aspecto tributário e fiscal, como na busca de estratégias de competitividade de mercado. Segundo este gestor, os avanços são diversos para as cooperativas integrantes, não só no quesito direto da redução de custos e melhor poder de barganha como desempenho estratégico, maior penetração de produtos e posicionamento de mercado.

Neste contexto a cooperativa estudada, Coopervap, consegue se estabelecer como referência regional com faturamento anual de mais de 180 milhões de reais, o quadro social é formado por mais de dois mil associados, a cooperativa emprega 500 funcionários diretos e é fonte de renda para o estado e município, gerando 
mais de 22 milhões de reais em Tributos e encargos sociais. Além disso, após mais de 50 anos de atuação a Coopervap tem um faturamento anual superior a $R \$$ 256.000.000,00, estes números corroboram com as informações expostas por BARROS (2006), reforçando a cadeia produtiva, além da geração de emprego e renda e a influência direta no desempenho financeiro do país.

As alianças estratégicas promovem melhor implantação, implementação e execução dos empreendimentos que estão voltados à geração de valor na cadeia do agronegócio, contribuindo desta maneira para a intercooperação. Assim, FAÇANHA (2012) pontua que as alianças estratégicas trazem como resultado as organizações integrantes se tornarem referência entre empreendimentos sustentáveis e com grande competência para produzir oportunidades econômicas. Os dados apresentados sobre a Cooperavap e CCGC reforçam esta posição da autora e atestam a credibilidade e possibilidade de sucesso em alianças estratégicas organizadas e estruturadas.

\section{CONCLUSÃO}

A aliança estratégica entre cooperativas do estado de Minas Gerais, como empreendimento cooperativo formal, foi motivada, principalmente, pela necessidade de respostas aos estímulos empresariais da atualidade como forma de manter vantagens competitivas de cada um dos participantes por meio da otimização dos custos e sinergia com outros parceiros que possuem algum diferencial de mercado. Outro fator motivador é a busca pela superação de entraves e também o aproveitamento de oportunidade que de maneira individual seria impossível de ser alcançada.

Também, a aliança estratégica afetou de forma positiva com referência a competitividade maior em negociar bens de produção e melhores preços dos produtos Coopervap no mercado. O que afeta negativamente são alguns aspectos culturais diferentes entre as integrantes, principalmente no comportamento e gestão das pessoas envolvidas. Entretanto, a colaboração entre as cooperativas é constante, principalmente no fator relativo à troca de conhecimentos relacionados às medidas legais tanto no aspecto tributário e fiscal, como na busca de estratégias de competitividade. Identifica-se que os avanços são diversos para as cooperativas integrantes, pois além da redução de custos, existe um melhor poder de barganha como desempenho estratégico, maior penetração de produtos e posicionamento de mercado.

As cooperativas participantes do Consórcio Cooperativo Grupo Cemil CCGC estão conseguindo obter resultados satisfatórios, mesmo com o pouco espaço de tempo e a estrutura organizacional enxuta. A posição apresentada pelo Gestor da Coopervap é que os resultados financeiros alcançados reforçam a importância do desenvolvimento de parcerias com vistas a um melhor desempenho no mercado. A aliança permite avanços não só no campo econômico, como também nos âmbitos da estratégia e da gestão, uma vez que consiste em um excelente espaço para troca de experiências e conhecimento, com grande influência para o desempenho organizacional como um todo.

A redução dos preços alcançada pela compra conjunta de produtos e insumos não interfere no desenvolvimento de ações de cada organização, mas interfere diretamente na lucratividade, onde são comprados por preços mais competitivos devido ao grande volume nas negociações. Ações como esta demonstram a possibilidade real no desenvolvimento de alianças estratégicas ao conseguirem melhor desempenho econômico. 
Em síntese, o CCGC tem alcançado seus objetivos ao longo dos três anos de sua implantação e conforme com a visão apresentada por uma das cooperativas participantes, vários benefícios são advindos desta parceria. Este consórcio, apesar de novo e com estrutura administrativa enxuta, consiste em um interessante exemplo de aliança estratégica de sucesso. Sugere-se para pesquisas posteriores o aprofundamento sobre o desenvolvimento deste consórcio cooperativo, bem como o posicionamento de outras cooperativas participantes a respeito do empreendimento.

\section{AGRADECIMENTOS}

Os autores agradecem aos gestores da cooperativa analisada pela colaboração com o estudo de caso. Também, à Fundação de Amparo à Pesquisa do Estado de Goiás - FAPEG, pela contribuição para a realização da pesquisa.

\section{REFERÊNCIAS}

ABBADE, E. B. Análise de Alianças Estratégicas sob a Ótica da Teoria dos Jogos. Revista de Administração da UNIMEP, v. 7, n. 3, 2009. Disponível em: < http://dx.doi.org/10.15600/1679-5350/rau.v7n3p24-45>. DOI: 10.15600/16795350/rau.v7n3p24-45.

BARDIN, L. Análise de Conteúdo. 5ed. Lisboa: Edições 70, 2009.

BARROS, G. S. C. Agronegócio brasileiro: perspectivas, desafios e uma agenda para seu desenvolvimento. Piracicaba, jul. 2006. 52 p. Disponível em: $<$ http://www.cepea.esalq.usp.br/especialagro/EspecialAgroCepea_all.doc>. Acesso em: 20 jul. 2016.

FAÇANHA, S. L. O. Aquisições, Fusões e Alianças Estratégicas na Configuração da Cadeia Sucroenergética Brasileira. 2012. 330 f. Tese (Doutorado em Administração) - Faculdade de Economia, Administração e Contabilidade, Universidade de São Paulo, São Paulo, 2012.

FERREIRA, M. A. M.; BRAGA, M. J. Diversificação e competitividade nas cooperativas agropecuárias. Revista de Administração Contemporânea, v. 8 n. 4 , p.33-55, 2004. Disponível em: <http://dx.doi.org/10.1590/S141565552004000400003>. DOI: 10.1590/S1415- 65552004000400003.

LAGO, A. Fatores condicionantes do desenvolvimento de relacionamentos intercooperativos no cooperativismo agropecuário. 2009. $179 \mathrm{f}$. Tese (Doutorado em Agronegócios) - Universidade Federal do Rio Grande do Sul, Porto Alegre, 2009.

LORANGE, P.; ROOS, J. Alianças estratégicas: formação, implementação e evolução. São Paulo: Atlas, 1996.

PELEGRINI, D. F.; SHIKI, S. F. N.; SHIKI, S. Uma abordagem teórica sobre cooperativismo e associativismo no Brasil. Extensio: R. Eletr. de Extensão, v. 12, n. 19, p. 70-85, 2015. Disponível em: $<$ https://periodicos.ufsc.br/index.php/extensio/article/download/1807-

0221.2015v12n19p70/30468>. DOI: http://dx.doi.org/10.5007/1807$0221.2015 \mathrm{v} 12 \mathrm{n} 19 \mathrm{p} 70$. 
SILVA, G. J. C.; SOUZA, E. C.; MARTINS, H. E. P. Produção agropecuária em municípios de Minas Gerais (1996-2006): padrões de distribuição, especialização e associação espacial. Revista de Economia e Sociologia Rural, v. 50, n. 2, p. 333 349, 2012. Disponível em: <http://dx.doi.org/10.1590/S0103-20032012000200008>. DOI: 10.1590/S0103-20032012000200008.

SOUZA, D. B.; BALDIN, C. O papel das alianças estratégicas na competitividade das cooperativas de leite. Organizações Rurais e Agroindustriais, v. 7, n. 3, p. 324334, 2006. Disponível em: < http://repositorio.ufla.br/handle/1/9121>.

SOUZA, E. C. Dinâmica Recente do Setor Agropecuário de Minas Gerais: Uma Análise Econométrica Espacial. Monografia de Graduação: Instituto de Economia (Universidade Federal de Uberlândia), p. 66. 2010. 